\title{
Blind Hurdle Stick: Android Integrated Voice Based Intimation via GPS with Panic Alert System
}

\author{
Vani Walvekar, Swathi T Shetty*, Shruthi N,Yashaswini B R \\ Department of CSE, GSSSIETW, Mysuru, Karnataka, India
}

DOI: https://doi.org/10.21467/proceedings.1.12

* Corresponding author email: swathitshetty88@gmail.com

\begin{abstract}
The acknowledgment of the fact that it is so hard to do things when our eyes are shut urged us to think of an inventive thought. There are roughly 36 million individuals over the world who are visually impaired and more than 12 million are from India.Till date blind people are managing to travel with walking stick and obstacle detecting cane and other devices.As of late there has been a great deal of Electronic Travel Aids (ETA) plans and these devised to help the blind navigate independently and safely.In this paper, smart stick acts like an artificial vision and alarm unit that enables visually impaired people to find difficulties in detecting obstacles. This framework utilizes sensors for hurdle detection, bluetooth alongside an android application which give a voice help to the visually impaired individual. To identify position of the blind person a GPS module with a panic alert system is integrated so that intended person can be notified.This system has an advanced feature integrated to help the blind find their stick if it is misplaced.
\end{abstract}

Keywords-Smartstick, Ultrasonicsensor, GPS, Microcontroller.

\section{INTRODUCTION}

Physical movement is a test for outwardly weakened people, since it can wind up precarious to recognize where he is, and how to get where he needs to travel between various places. To explore obscure spots, he will bring a located relative or his companion for help. Existing devices are able to detect and recognize objects that emerge on the floor, but a considerable risk also includes the objects that are at a sudden depth, or obstacles above waist level or stairs. Subsequently we were inspired to build up a brilliant stick to defeat these constraints. The most widely recognized instrument that the visually impaired right now uses to explore is the standard white stick. In the past different systems are designed with limitations without a solid understanding of the non-visual perception. A portion of the frameworks are just for indoor routes and has no obstacle recognition and deciding area highlighted in open air condition.

However, in contrast with different advancements numerous visually impaired direction frameworks utilize ultrasound, as a result of its invulnerability to the ecological commotion. Another motivation behind why ultrasonic is prevalent is that the innovation is generally cheap, and furthermore ultrasound producers and finders are sufficiently little to be conveyed

(C) 2018 Copyright held by the author(s). Published by AIJR Publisher in Proceedings of the $3^{\text {rd }}$ National Conference on Image Processing, Computing, Communication, Networking and Data Analytics (NCICCNDA 2018), April 28, 2018.

This is an open access article under Creative Commons Attribution-NonCommercial 4.0 International (CC BY-NC 4.0) license, which permits any non-commercial use, distribution, adaptation, and reproduction in any medium, as long as the original work is properly cited. ISBN: $978-81-936820-0-5$ 
Blind Hurdle Stick: Android Integrated Voice Based Intimation via GPS with Panic Alert System

without the requirement for complex hardware. Apart from the conventional navigation systems, a blind aid systems can be provided a new dimension of real-time assistance and artificial vision alongwith dedicated obstacle detection circuitry. This different units are discussed to implement the design of a 'Smart stick' for blind. Our paper aims to design and implement an intelligent and inexpensive stick with Global Positioning System (GPS) for the visually impaired peoples, which will detect the obstacle and hurdle in the path and also determine the position and location through GPS coordinates. The implemented model approximately detects the hurdle upto $50 \mathrm{~cm}$ and intimates the user through voice.It also determines the position and location by sending the latituide and longitude of the user to the intended person via sms.

\section{II LITERATURE SURVEY}

[1] The improvement of an Electronic Traveling Aid (ETA) unit to enable the visually impaired individuals to discover hindrance to free way. This ETA is settled to the stick of the visually impaired individuals. At the point when the question is distinguished close to the blinds stick it cautions them with the assistance of vibratory circuit (speakers or earphones). The area of the visually impaired is discovered by utilizing Global System for Mobile interchanges (GSM) and Global Position System [2]. Here reflected signals received from the barrier objects are used as inputs to PIC microcontroller. The microcontroller is then used to determine the direction and distance of the objects around the blind. It also controls the peripheral components that alert the user about obstacle's shape, material, and direction. The implemented system is cheap, fast, and easy to use and an innovative affordable solution to blind and visually impaired people in third world countries.the paper exhibits a hypothetical model and a framework idea to give a shrewd electronic guide to blind individuals. The framework is planned to give general measures - Artificial vision and question discovery, continuous help through worldwide situating system(GPS). The framework comprise of the sensors

such as ultrasonic sensors, GPS module, stereo cameras and double input framework soundrelated and additionally vibratory circuit.[3]The smart white cane uses the ultrasonic sensors arranged in such a way that it detects pits, potholes, downfalls, a staircase ( up and down), low lying and knee level obstacles and even those above the waist.Theuser is notified about the same by the pre-recorded sound messages and a haptic feedback in form of vibrations. This can considerably alleviate the risk of the user injuring himself.[7]An overview of theprototype, design issues, and its different modules which integrate GPS and Wi-Fi localisation with aGIS, passive RFID tags, and computer vision. The prototype addresses global navigation for going tosome destiny, by following known landmarks stored in the GIS in combination with path optimisation, and local navigation with path and obstacle detection just beyond the reach of the white cane. Thesystem does not replace the white cane but complements it, in order to alert the user to loominghazards. In addition, computer vision is used to identify objects on 
shelves, for example in a pantry orrefrigerator. The user-friendly interface consists of a fourbutton hand-held box, a vibration actuatorin the handle of the white cane, and speech synthesis [8]. The visually impaired individual can be guided through the voice caution about the obstacles detected. It depicts a way to deal with help blind individuals in distinguishing the nearness and closeness of impediments in their way by methods for Ultrasonic waves which is utilized to discover the obstruction, if discovered it is intended to the individual through the particular vibration engine and headset. A GPS route gadget can help explore turn-by-turn bearings. With it, you can achieve any goal you need no sweat, without requiring persons [9]. A trial framework for the transformation of pictures into sound examples was intended to give sound-related picture portrayals inside a portion of the known impediments of the human hearing frameworks potentially as a stage towards the improvement of a dream substitution gadget for the visually impaired. The utilization of an invertible (balanced) picture to-sound mapping guarantees the safeguarding of visual information. [10] A wearable equipment consists of head hat and mini hand stick to help the blind person to navigate alone safely and to avoid any obstacles that may be encountered, whether fixed or mobile, to prevent any possible accident. The main component of this system is the infrared sensor which is used to scan a predetermined area around blind by emitting-reflecting waves. [11] Thestrategy for deciding separation utilizing a stereo coordinating technique to help blind people for their route. An enhanced territory based stereo coordinating is performed over the changed pictures to compute thick difference picture. Low surface channel and left/right consistency look at are conveyed to expel the clamors and to feature the hindrances. A sonification technique is proposed to outline dissimilarity picture to stereo melodic sound, which has data about the highlights of the scene before the client. The sound is passed on to the visually impaired client through stereo headphones. [12] The system uses state of the art deep learning techniques from Microsoft Cognitive Services for image classification and tagging. The Experience is powered by voice assistant 'Alexa' through Amazon Echo. This System aims bring the beautifulworld as a narrative to the visually impaired. The main module is of raspberry pi which is on its own a mini-computer, which processes the image captured by the webcam. The Image is converted into Text using AWS Dynamo DB Which is one type of the software used which is a fully managed.NoSQL database service that provides fast and predictable performance with seamless scalability. When the blind people request that Amazon echo to describe the scene, then the Alexa Skills Kit triggers Amazon Lambdafunction to bring the data from the database DynamoDB. The AWS Lambda Function is used to convert text into speech. The correct text is the played as an audio on the Alexa device.

\section{PROPOSED METHOD}

The proposed framework intends to give a straight forward and viable course to help blind which gives a feeling of fake vision by giving information about the environment situation of

Proceedings of the $3^{\text {rd }}$ National Conference on Image Processing, Computing, Communication, Networking and Data Analytics (NCICCNDA 2018) 
Blind Hurdle Stick: Android Integrated Voice Based Intimation via GPS with Panic Alert System

static and dynamic question around them. The system can be intended to take type of a separable and convenient gadget, which can be genuinely mounted on a basic outwardly disabled stick or visually impaired stick. This requires an unmistakable vision of the coveted framework objectives. Different framework parameters are in this manner should have been assessed to outline all intents and purposes of the system. Our proposed system mainly uses ultrasonic sensors to detect the obstacles ahead utilizing ultrasonic waves. On detecting obstacles the sensor passes this information to the microcontroller. The microcontroller at that point forms this information and figures if the impediment is sufficiently close. On the off chance that the impediment isn't that nearby the circuit does nothing. On the off chance that the impediment is sufficiently close then the microcontroller sends a content to smart phone which inturn is changed over to speech. Our venture is an inventive thought of wise framework which has fundamentally two highlights the first is Obstacle identification and the second one is giving exact area and position through GPS. It will give security and support to outwardly disabled people. The ultrasonic sensors in the framework will detect encompassing and will distinguish the impediments and offer criticism to discourse IC.

\section{METHODOLOGY}

The design of the smart stick distantlt $\backslash y$ consists of

1.Microcontroller(Arduino Mega)

2.Obstacle detection unit

3.Bluetoothmodule link with mobile(GPS and Android)

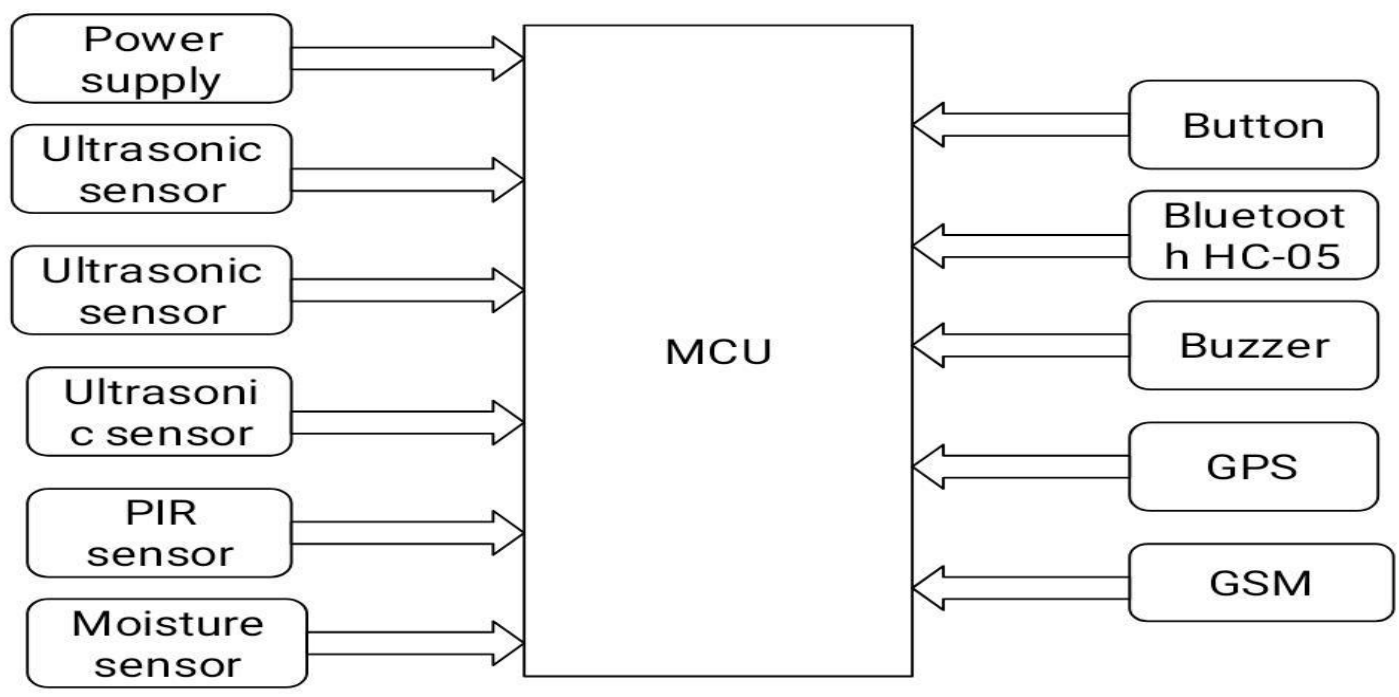

Fig 4.1: Transmitting End

The power supply activates the circuit. The sensor transmitter transmits the frequency, which reflects from the obstacle. Sensor receiver receives the reflected frequency and gives it to 
microcontroller. The microcontroller processes it and gives signal to speech IC. Speech IC gives sound and start to inform the person that the obstacle is detected through headphone. The GPS module receives the coordinate continuously and gives it to the microcontroller. Microcontroller access the location from the GPS modem and transmit the location to the GSM modem which will send a SMS messages to the all saved number.

The figure below portrays the proposed sysem of an integrated blind stick. The design parts involve different subsystems.

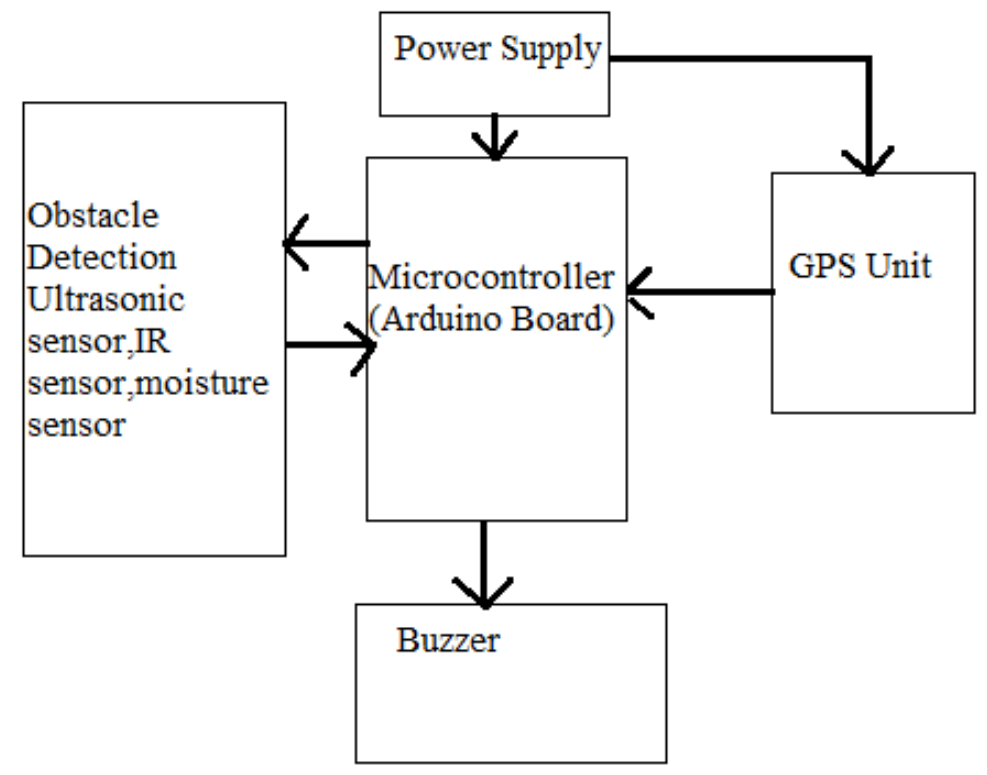

Fig 4.2 : Working model of smart stick

\subsection{Microcontroller (Arduino Mega)}

Key reason for selection of the Mega 2560 ,it has 54 digital input/output pins (of which 15 can be used as PWM outputs), 16 analog inputs, 4 UARTs (hardware serial ports), a $16 \mathrm{MHz}$ crystal oscillator, a USB connection, a power jack, an ICSP header, and a reset button.Port 2.0 pin to Left ultrasonic sensor,port 3.0 pin to front ultrasonic sensor and Port4.0 for Right ultrasonic sensor for reception of trigger and echo in case of obstacle detection .Panic Button in conditions of distress is connected to Port6.0.Port 5.0 is connected to passive infrared sensor and port 7.0 is connected to moisture sensor.Software used for microcontroller programming is Arduino 1.8.5 with embedded C language.

\subsection{The Obstacle detection unit-}

It consists of three Ultrasonic sensors, PIRsensor and a Moisture sensor. Ultrasonic sensor uses $40 \mathrm{KHz}$ signals to detect obstacle up to $50 \mathrm{~cm}$ and on detection based on speed of sound waves, and then will send Binary serial signal on Rx Pin of Microcontroller. Based on different

Proceedings of the $3^{\text {rd }}$ National Conference on Image Processing, Computing, Communication, Networking and Data Analytics (NCICCNDA 2018) 
Blind Hurdle Stick: Android Integrated Voice Based Intimation via GPS with Panic Alert System

distances the buzzer will ring for different durations. The PIR sensors use Infrared beam to sense surrounding objects and on reception send perceived data to Microcontroller. The range of left and right sensors can be increased but for proposing model we prefer to keep system simple so that it is easily implementable and conceptually easy to understand. Moisture sensor is used to detect moisture infront of the blind person.Moisture sensor consists of two plates where conductivity is produced inbetween. As water is high conductor of electricity it produces conductivity between plates and the microcontroller receives the data from sensor.Microcontroller sends appropriate message to the smart phone and then it is converted to voice message to alert about the moisture infront of the blind person.

Hurdle Recognition

\section{Direction}

Sensor Used

Distance Range/State

\begin{tabular}{llll}
\hline \multirow{2}{*}{ Front } & Ultrasonic & Range & $50 \mathrm{~cm}$ \\
& Sensor & & \\
\hline \multirow{2}{*}{ Right } & Ultrasonic & Range & $50 \mathrm{~cm}$ \\
& Sensor & & \\
\hline \multirow{2}{*}{ Left } & Ultrasonic & Range & $50 \mathrm{~cm}$ \\
& Sensor & & \\
\hline & Passive infrared & PIRStatus & Low \\
& Sensor & & \\
\hline & Moisture sensor & liquidState & Low \\
\hline
\end{tabular}

\subsection{Bluetooth Module to Mobile Link}

HC05 model is used. Bluetooth is mainly used to send the data from microcontroller to the smart phone. In case of panic situations supposing the blind person has some difficulty in finding his location, button provided within the smart stick will trigger the mobile to send a SMS to registered mobile numbers with exact GPS location of the person.

\section{EXPERIMENTAL RESULT}

The development of smart stick utiliziesArdunio mega 2560 and smart phone. The ultrasonic is function to send the transmitted pulse and feedback the reciver pulse to determine the distance in front.The function of smart phone is to feed warning back, in the form of voice message and buzzer, to users. Firstly, if there is an obstacle or barrier in front, right and left ultrasonic sensors will send the signal to circuit microcontroller. The first case is when the obstacle is in the right direction of the blind, then the user will be intemated by smart phone using application.The second case is when theobstacle is at the left side of the blind, then the 
user will be intimated by smart phone using application. The third case is when the obstacle is in front of the blind, then the user will be intemated by smart phone using application. The fourth case is when the user misplaces the stick, user will be able to lacate the misplaced stick using passive infrared sensor.The fifth case is when moisture in detected infront of user, then it will intimate with the help of moisture sensor installed at bottom of blind stick.the sixth case is an advanced feature using which user will be able to send his/her present location in case of emergency.

\section{CONCLUSION}

With the proposed architecture, the visually impaired individuals can move starting with one place then onto the next without others help, which prompts increment self-govenance for the visually impaired. The created model gives great outcomes in identifying obstacles placed at distance in front of the user. In the meantime, worldwide, situating framework (GPS) can be connected with the stick for locatingthe current position, so intended guardian can know user's present position in case of emergency.

\section{REFERENCES}

[1] Calder, David J,Curtin, "An obstacle signaling sytem for the blind,Digital Ecosystems and Technologies,”Confrence(DEST),2011 Proceedings of the 5 th IEEE International Confrence on 30 June 2011 .

[2] Shruti Dambgare , Prof.A.Sakhare,"Smart Stick for Blind:ObstacleDection,Artificial Vision and Real- Time assistance via GPS" Confrence (NCICT)2011, Proceedings 2nd National Confrence on Information and CommunicationTechnology 2011.

[3] Rohith seth "Smart White Cane- an elegant and economic walking aid".

[4] World Health Organization, "Visual Impairment and Blindness," Fact sheet N “282”, Oct 2014.

[5] National Disability Policy: A Progress Report - October 2014, National Council on Disability, Oct 2014.

[6] T. Terlau and W. M. Penrod, "K'Sonar Curriculum Handbook", Available from: "http://www.aph.org/manuals/ksonar.pdf", June 2008 [4] L. Whitney, "Smart cane to help blind navigate", Available from: "http://news.cnet.com/8301-17938\_105-10302499-1.html", 2009.

[7] J.M. Hans du Buf, J.Barroso, Jojo M.F. Rodrigues, H.Paredes, M.Farrajota, H.Fernandes, J.Jos, V.Teixeira, M.Saleiro.”The SmartVision Navigation Prototype for Blind Users”. International Journal of Digital Content Technology and its Applications, Vol.5 No .5, pp. 351 - 361, May 2011.

[8] I. Ulrich, and J. Borenstein, "The guide cane-Applying mobile robot technologies to assist the visually impaired," IEEE Transaction on Systems, Man, and Cybernetics-Part A: Systems and Humans, vol. 31, no. 2, pp. 131-136, 2001.

[9] P. Meijer, "An Experimental System for Auditory Image Representations," IEEE Transactions on Biomedical Engineering, vol.39, no 2, pp. 112-121, Feb 1992.

[10] M. Nie, J. Ren, Z. Li et al., "SoundView: an auditory guidance system based on environment understanding for the visually impaired people," in Proceedings of the 31st Annual International Conference of the IEEE Engineering in Medicine and Biology Society: Engineering the Future of Biomedicine (EMBC '09), pp.7240-7243, IEEE, September 2009.

[11] G. Balakrishnan, G. Sainarayanan, R. Nagarajan and S. Yaacob, "Wearable Real-Time Stereo Vision for the Visually Impaired," Engineering Letters, vol. 14, no. 2, 2007.

[12] G. P. Fajarnes, L. Dunai, V. S. Praderas and I. Dunai, "CASBLiP- a new cognitive object detection and orientation system for impaired people," Proceedings of the 4th International Conference on Cognitive Systems, ETH Zurich, Switzerland, 2010.

Proceedings of the $3^{\text {rd }}$ National Conference on Image Processing, Computing, Communication, Networking and Data Analytics (NCICCNDA 2018) 